\title{
Capabilities of automated LA-ICP-TOFMS imaging of geological samples $\dagger$
}

Cite this: J. Anal. At. Spectrom., 2020 35,2255

\author{
Christoph Neff, (D) a Peter Keresztes Schmidt, (D) a Paolo S. Garofalo, ${ }^{b}$ \\ Gunnar Schwarz (D)*a and Detlef Günther (D)*a
}

Element imaging aims to provide quantitative data on multi-element distributions from major to trace elements with high lateral resolution. Here, we describe a control system for laser ablation inductively coupled plasma time-of-flight mass spectrometry (LA-ICP-TOFMS) imaging with complex integration of translational stage, laser, and mass spectrometer data acquisition which was tested on glass and mineral samples. In particular, besides single pulse imaging at a laser repetition rate of $100 \mathrm{~Hz}$ using a $5 \mu \mathrm{m}$ laser spot size, a hole drilling imaging approach provided higher pixel sensitivity and lower limits of detection ( $<1 \mathrm{mg} \mathrm{kg}^{-1}$ for most heavy elements) while maintaining the same lateral resolution. An optional surface cleaning pulse can be applied without additional recording of data. Furthermore, the ablation area can be adapted to specific object structures of interest and leads to significant shorter imaging times. Triggering the data acquisition for every ablation position led to binned pixel data in relation to the sample position. This simplifies the data evaluation and allows a more automated image generation. The approach presented in this study enables flexible adjustments of distinct ablation modes to a specific analytical task and provides the basis for fully automated element imaging. To test the applicability of our approach, two complex geological samples containing crystalline solids were imaged to gain insights into the distribution of trace elements that occur typically in the low $\mathrm{mg} \mathrm{kg}^{-1}$ range. We show that both single pulse and hole drilling ablation modes allow the determination of a large number of trace elements. However, the hole drilling mode shows a superior sensitivity per pixel, which in turn provides more detailed information about the formation of geological samples.

Received 18th May 2020

Accepted 23rd July 2020

DOI: $10.1039 / \mathrm{dOja00238k}$

rsc.li/jaas distribution of coexisting minerals during metamorphism [e.g., ref. 5] to name a few. Most of these studies were conducted using bulk analyses of individual minerals by ancillary mass spectrometric techniques, or with spot analyses carried out via electron probe micro analysis. The broad range of small-scale processes revealed with these methods lead to the use of element distribution within minerals as monitors of largerscale, more general geological processes.

Technical advances in the determination of major and trace element distributions in key rock minerals improved the definition of the large-scale geological processes significantly. For instance, refined definition of growth conditions and stability intervals of the garnets and zircons groups of silicate minerals allowed improved constraints on rock metamorphic processes ${ }^{6,7}$ and the discrimination of the genesis of ore minerals in economic deposits. ${ }^{8}$ These advances demonstrated invariably that high resolution techniques are at the heart of detailed investigations of geological samples. This calls for high spatial resolution, which is essential to distinguish small geometrical features within samples, and for a combination of high sensitivity with low limits of detection (LOD), and simultaneous multi-element detection capability from major to trace elements.
${ }^{a}$ Laboratory of Inorganic Chemistry, Department of Chemistry and Applied Biosciences, ETH Zurich, Vladimir-Prelog-Weg 1, 8093 Zürich, Switzerland. E-mail: detlef. guenther@sl.ethz.ch; schwarz@inorg.chem.ethz.ch

${ }^{b}$ Università degli Studi di Bologna, Dipartimento di Scienze Biologiche, Geologiche ed Ambientali, Via Zamboni 67, 40126 Bologna, Italy

$\dagger$ Electronic supplementary information (ESI) available. See DOI: 10.1039/d0ja00238k 
Since the advent of laser ablation inductively coupled plasma mass spectrometry (LA-ICP-MS), ${ }^{\mathbf{9}, 10}$ methods were developed to determine the bulk composition of particular phases by spot analysis, ${ }^{\mathbf{1 1}}$ the element distribution in one dimension (line scan), ${ }^{12-17}$ of two-dimensional areas, ${ }^{18-22}$ and even spatial spread (3D). ${ }^{\mathbf{2 3 2 4}}$ The recent revival of ICP-TOFMS and low dispersion laser ablation cells have led to a rise of interest in fast, multielement, and high spatial resolution LA-ICP-MS imaging for geological samples..$^{\mathbf{2 0 2 4 - 2 6}}$ ICP-TOFMS mass analyzers provide quasi-simultaneous detection of the full mass spectrum with fast acquisition rate. ${ }^{27}$ Altogether, small laser spot sizes, different ablation cells and fast, simultaneous multi-element detection with ICP-TOFMS offer a unique and promising combination to tackle the analytical challenges of element imaging.

High lateral resolution can be achieved by a small laser ablation spot size. Imaging has been achieved at sub-cellular resolution using a laser spot size of $\leq 1 \mu \mathrm{m} .^{22,28,29}$ In some cases, simple laser scan distance in relation to the data acquisition frequency was misunderstood as lateral resolution(e.g. ref. 30) However, while sensitivities still need to be sufficient to yield signals for individual laser pulses, smaller pixel sizes were also accomplished with overlapping scans and signal deconvolution. ${ }^{31-35}$

To achieve high data acquisition speed while maintaining the lateral resolution, low dispersion ablation cells have to be used to transport the aerosol in a dense plume without pulse-topulse aerosol mixing into the ICP-MS. ${ }^{36}$ Shorter signal durations increase the signal-to-noise ratio and allows a faster data acquisition. In recent years, signal duration of $<10 \mathrm{~ms}$ (full width at $1 \%$ of the maximum, FW0.01M) for LA-ICP-MS imaging were achieved..$^{\mathbf{2 0} 28,37,38}$ These ablation cells deliver signal durations between 4 and 9 ms (FW0.01M). As an early benchmark, Tanner and Günther ${ }^{39}$ achieved signal durations in the range of 1-3 ms for an in-torch ablation approach, although without the capability to perform imaging experiments. Similar short signals were most recently reported by Van Malderen et al. ${ }^{29}$ for imaging applications.

As noted earlier by Paul et al. ${ }^{40}$ software development for LAICP-MS imaging is lagging behind compared to other imaging techniques. A detailed discussion of this issue can be found in a review by Weiskirchen et al. ${ }^{41}$ Until now, the major focus was on software solutions for data analysis. ${ }^{\mathbf{4 0 - 4 6}}$ Nevertheless, the more LA-ICP-TOFMS imaging becomes routine, the more need there is for an automation of the data acquisition process. In addition, there is hitherto mostly unused potential for utilizing the capabilities of low dispersion ablation cells in combination with LA-ICP-TOFMS for convenient data acquisition and processing, which can support different aims for imaging beyond mere line scan approaches. For instance, in contrast to quadrupole MS and sector field MS, TOFMS provides complete mass spectra and the full information on isotope signals can be accessed for each laser pulse. Once data is stored in bins representing each ablation position the cumbersome isolation and sectioning of the signals can be avoided, accelerates data processing $^{24}$ and offers exceptional opportunities for data analysis, e.g. phase specific pixel clustering and thereby improved quantitative data even for small-size sample features. ${ }^{25,26}$ Moreover, continuous line scans with overlapping laser spots distort spatial information since the ablated material from individual laser pulses originate from different depths along the scan direction. The TOFMS timing can be adjusted so that data for a single or multiple laser pulses directed at the same sample position without sample stage movement can be stored in a single data bin. Hence, software which integrates control of sample translation stage, laser, and ICP-TOFMS is required to further foster LA-ICP-TOFMS imaging with different ablation modes fitting to different study requirements.

Here, we present an integral control system for flexible LAICP-TOFMS imaging and show how such a system can be applied to the determination of element distributions in geological samples. We report a leap towards LA-ICP-TOFMS imaging automation and applied different ablation modes by synchronization of translational stage, the laser, and mass spectrometer data acquisition with a custom-built imaging control system. In particular, the setup enables to perform a single pulse imaging mode with high scanning speeds or a hole drilling imaging mode that provides lower LODs. In the former case, laser pulses are applied in quick succession in an edge-to-edge raster over the area of interest. For analysis of elements at lower mass fractions, multiple laser pulses per sample position were applied and thereby the pixel LOD improved while the same lateral resolution was maintained. Due to synchronized and selective triggering in both modes, data is stored for each pixel individually, which assist analysis, data processing, and image generation. The application of these imaging modes to two geological samples demonstrate that they can be employed strategically for the analysis of mineral samples.

\section{Experimental}

\section{Instrumentation}

For all studies, an argon-fluoride (ArF) excimer LA system (193 nm, GeoLas C, Lambda Physik, Goettingen, Germany) in combination with a modified ${ }^{47}$ low dispersion ablation cell based on the tube cell design ${ }^{\mathbf{2 0 , 2 8}}$ was coupled to an ICP-TOFMS instrument (icpTOF2R, Tofwerk AG, Switzerland) where the vacuum pump was replaced by an UNO 065 D pump (Pfeiffer, Asslar, Germany). LA was carried out in a helium atmosphere ${ }^{\mathbf{4 8}}$ (99.999\%, PanGas AG, Dagmersellen, Switzerland) and a stream of argon gas (99.996\%, PanGas AG, Dagmersellen, Switzerland) served as the carrier gas. The piezo-electrically driven $x y z$ translational stage (1 $\mathrm{nm}$ scan resolution, SmarAct $\mathrm{GmbH}$, Oldenburg, Germany), the laser and the TOFMS data acquisition were triggered using the custom-built imaging control system, which allows an integrated control of these three devices.

\section{Element imaging}

Typical LA and ICP operating conditions are provided in Table 1. For all experiments, a homogenized, circular laser beam was focused onto the sample surface. LA and the TOFMS data 
Table 1 Typical LA and ICP operating parameters

Laser fluence

Laser spot diameter

Ablation gas flow (He)

Carrier gas flow (Ar)

Auxiliary gas flow (Ar)

Cooling gas flow (Ar)

RF power

Notch filter

${ }^{232} \mathrm{Th}^{+} /{ }^{238} \mathrm{U}^{+}$

$\mathrm{ThO}^{+} / \mathrm{Th}^{+}$

\author{
$15 \mathrm{~J} \mathrm{~cm}^{-2}$ \\ $5 \mu \mathrm{m}$ \\ 1.25-1.45 $\mathrm{L} \mathrm{min}^{-1}$ \\ $0.7-0.9 \mathrm{~L} \mathrm{~min}^{-1}$ \\ $0.8 \mathrm{~L} \mathrm{~min}^{-1}$ \\ $16 \mathrm{~L} \mathrm{~min}^{-1}$ \\ 1400-1550 W \\ ${ }^{40} \mathrm{Ar}^{+},{ }^{40} \mathrm{Ar}_{2}^{+},{ }^{56} \mathrm{Fe}^{+},{ }^{75} \mathrm{As}^{+}$ \\ $0.9-1.1$ \\ $<1 \%$
}

acquisition were synchronized using the external imaging control system. The data acquisition was triggered for every ablation position. 9-10 data points were measured containing each the full mass spectrum for each laser sampling position.

To generate consecutive line scans in single pulse (SP) mode, a laser repetition rate of $100 \mathrm{~Hz}$ and a continuous translational sample moving speed of $500 \mu \mathrm{m} \mathrm{s}^{-1}$ were applied, leading to an edge-to-edge placement of the ablation spots. The overlapping line scan (OL) mode was carried out using a laser repetition rate of $100 \mathrm{~Hz}$ and a continuous translational sample moving speed of $20 \mu \mathrm{m} \mathrm{s}^{-1}$. This led to 25 laser pulses per $5 \mu \mathrm{m}$ sample movement. Consecutive lines were aligned edge-to-edge. For the hole drilling (HD) mode, 25 laser pulses were fired on the same sample position before the sample was moved to the next position. The signal of 25 laser pulses was summed and displayed as a square pixel of $5 \mu \mathrm{m} \times 5 \mu \mathrm{m}$ for the OL and HD mode. In the hole drilling mode with cleaning pulse ( $\mathrm{CP}+\mathrm{HD})$, a cleaning pulse was employed at each sample position prior to the start of the hole drilling. There was no ICP-MS data recorded for the cleaning pulse. The triggering pattern and the corresponding TOFMS data acquired of the four ablation modes SP, OL, HD, and CP + HD are illustrated in Fig. 1. The imaging of the minerals ilvaite, hedenbergite, quartz and the analysis of the corresponding external reference material were conducted with additional notch filters for the major elements ${ }^{56} \mathrm{Fe}$ and ${ }^{75}$ As to avoid detector saturation. Gas blank signals were acquired as separate files.

\section{Software development}

The software for the imaging control system was written considering the possibility of flexible adaptation and development. Due to its popularity in the scientific communities, its cross-platform compatibility, and the large library ecosystem available, Python 3 (https://python.com) was chosen as the main development language and wxPython (https:// wxpython.org) was selected as graphical user interface library with multi-platform compatibility. Furthermore, the software $(\approx 4000$ lines of code) was written in modules so that the hardware interfacing parts and the measurement routines can be exchanged or adapted independently from one another without the need to rewrite other parts.

The laser system was controlled using two separate communication channels. Using an ASCII protocol over a serial interface (RS-232) all parameters of the laser system are controlled (high voltage, power supply mode, discharge voltage, internal or external triggering mode, readout of status, and error messages). The linear

r.

Imaging Mode

Top view

Cross section

TOFMS data acquired
Single pulse
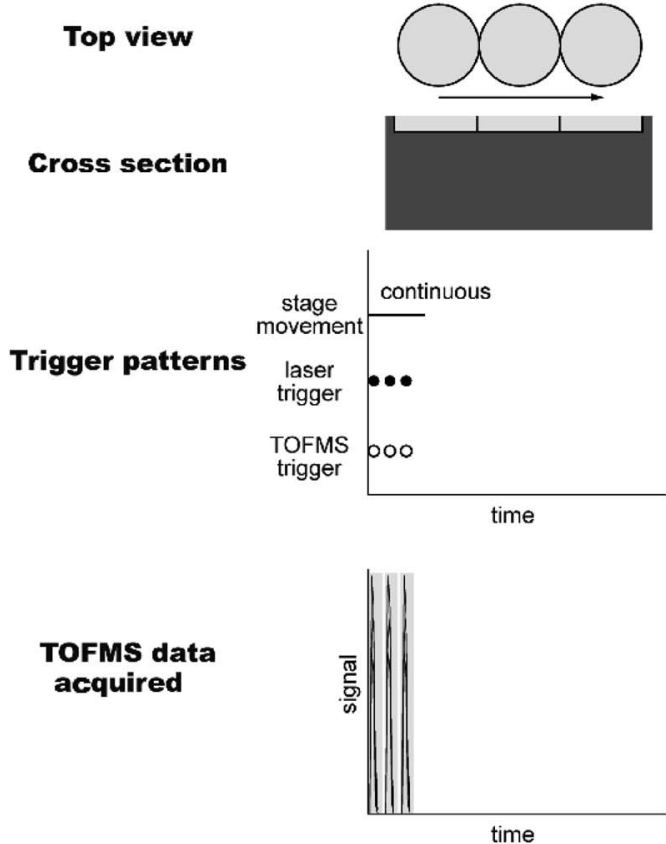

Hole drilling
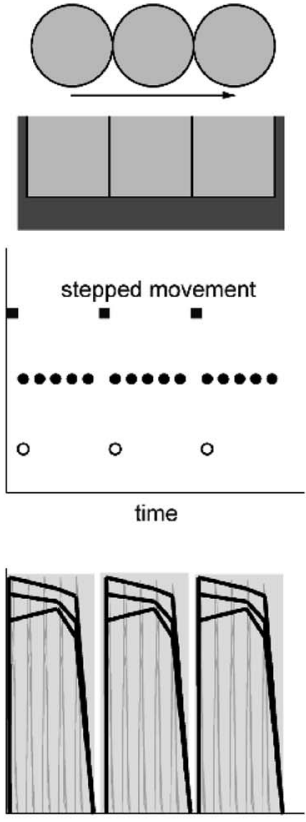

time
Hole drilling with cleaning pulse
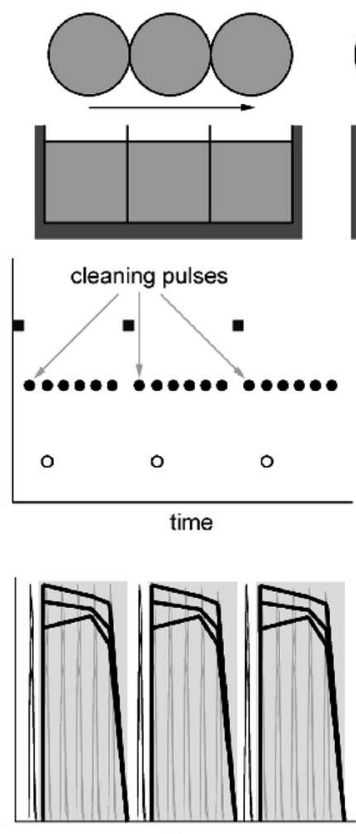

time

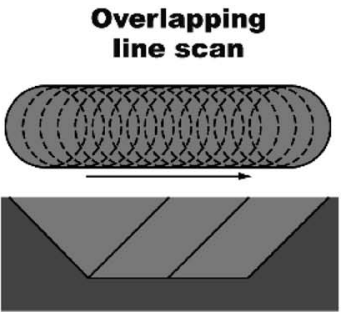

continuous

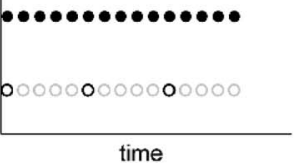

time

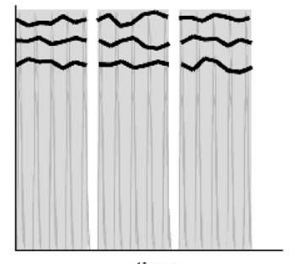

time

Fig. 1 Comparison of the ablation modes employed in this study for the acquisition of three pixels. The three modes to the right ablate more material. The acquired TOFMS data was binned for each pixel into different data sections, indicated as grey shadings in the bottom line. The signals resulting from cleaning pulses schematically shown are not recorded. Based on the longer integration duration in the OL mode, TOFMS trigger signals were intentionally left out to sum the signal of multiple laser pulses in one bin. 
piezo-electrical actuators of the sample translation stage were controlled via a MCS 3-axis controller (SmarAct, Oldenburg, Germany) using the integrated USB port with a proprietary binary protocol. A Python C Foreign Function Interface (CFFI) wrapper around the supplied C library was written for integration into the main program. For triggering the laser and TOFMS, a microcontroller (Arduino Uno, Arduino AG, Switzerland) was used and placed in-between. This avoids additional jitter of the trigger signals and ensures sufficient task frequency stability. Communication with the trigger controller was implemented using an ASCII based serial protocol.

The imaging software dovetails different hardware components for LA-ICP-TOFMS imaging experiments, namely control of the movement of the sample translation stage, triggering of laser pulses, and ICP-TOFMS data acquisition in a highly coordinated manner. It further allows for different ablation modes based on specific parameters set by the user, which included laser spot size, laser pulse frequency, and size dimensions of the region of interest (ROI), while the latter may be divided into subsections. Additional parameters included the application of cleaning pulses and time delays.

The specific use of the ICP-TOFMS data for imaging, the data structure, the data acquisition and the need of dividing the analyzed areas into subsection are described in detail in the ESI. $\dagger$ In brief, the different dimensions of the TOFMS data are used to trigger the TOFMS data acquisition for individual pixels and accordingly store data separately and avoid part of the previous cumbersome data processing. ${ }^{24,35}$ Since the software prompts triggers and controls for the sample translation stage, laser, and data acquisition independently, flexible stage movements and ablation modes are available. Irrespective of ablation mode and sample movement, the imaging control system enables to acquire the full mass spectrum and a transient signal for each sample position results in one pixel. Additionally, the distinct transient signal acquisition allowed to monitor element variations as a function of ablation depth. Notably, the core software and procedures are vendor agnostic and not limited to the LA-ICP-TOFMS hardware employed in this study.

Other laser systems or ICP-MS can be employed in the imaging control system whenever an external trigger input is available to which the trigger board can be connected. Additional development effort has to be made only if data communication with the laser or ICP-MS is necessary. Successful experiments have been conducted using an EX5 laser (GAM LASER, Inc., Orlando, USA) as well as the CyTOF system (Fluidigm, San Francisco, USA). Every stage system which supports a minimal set of features (mainly setting position and movement speed) and has a programming interface which can be called from Python, can be integrated. The "TEMAimaging" software of the imaging control system is provided under an open-source license (http://doi.org/10.5905/ethz-1007-269) and is hosted at https://github.com/ethz-tema/TEMAimaging.

\section{Samples}

Two geological samples RM2 and RM3, collected from the historic Torre di Rio Fe-ore deposit of the Island of Elba (Italy), were used for this study. They consist of well-crystallized silicate assemblages showing significant size and morphological variations at very small scales (crystal sizes: from few $\mathrm{cm}$ to $10 \mu \mathrm{m}$ or less), which were considered to be well suited to test different ablation modes. The two samples have been collected from the same ore body (excavation front) at a distance of about $35 \mathrm{~m}$ from each other, and all geological evidence indicate coeval formation of the mineral assemblages as a result of a hydrothermal event. This in turn suggests consistent mineral compositions in the two samples.

The sample RM3 is an aggregate of ilvaite (formula: $\mathrm{CaFe}_{3}$ $\mathrm{Si}_{2} \mathrm{O}_{8}(\mathrm{OH})$ ), hedenbergite (formula: $\mathrm{CaFeSi}_{2} \mathrm{O}_{6}$ ) and pyrite (formula: $\mathrm{FeS}_{2}$ ). The other sample RM2 is an aggregate of crystallized ilvaite and quartz (formula: $\mathrm{SiO}_{2}$ ). Prior to our study, the mineralogical and chemical compositions of the two samples have been determined using ancillary optical and electron probe microanalytical techniques (ESI Fig. S1 $\dagger$ ). The NIST SRM 610 was used as external reference. ${ }^{\mathbf{4 9 , 5 0}}$

\section{Data processing and analysis}

The binned TOFMS spectra were summed for each pixel using an in-house written script compiled on Python. The peak shape of the isotopes in the mass spectrum was adjusted according to Burger et $a .^{25}$ Furthermore, the baseline was subtracted, the mass calibration was adjusted and the isotope intensities were integrated using the data analysis package "Tofware" (version 2.5.11 http://www.tofwerk.com/tofware) running in "Igor Pro" (Wavemetrics, OR, USA), which allowed to obtain isotope intensities for individual pixels. Average gas blank signal intensities were subtracted and quantification of element mass fractions in the samples were performed for each pixel based on $100 \%$ mass normalization ${ }^{51}$ for 62 elements using NIST SRM 610 as an external reference material and assuming that all elements are present as oxides and all subsections were combined using another in-house written Python script.

The TOFMS data structure of "bufs" and "writes" was used as a relative coordinate system to create quantified element distribution maps (ESI $\dagger$ ). Data processing was automated for both Python scripts. LODs were calculated and plotted according to Poisson counting statistics ${ }^{52,53}$ using an in-house written script running on R. ${ }^{54,55}$

The TOFMS data acquisition is conducted without loss of information on mineral composition, and the simultaneous detection using a TOFMS allows measuring an extensive mass range $(\mathrm{m} / \mathrm{z} 23-238)$ in a given mineral without preselection of elements of interest. In order to carry out data analysis and interpretation, the determined element mass fractions can be exported as comma-separated values from the data analysis software and used with other software packages such as XMapTool. ${ }^{56}$

\section{Results and discussion}

The features of the software control system enable customized adaptation of the LA-ICP-TOFMS imaging experiment to the requirements of the sample and analytical challenge. Although 
other modes can be implemented, we studied two distinct ablation modes in greater detail. The results are discussed on mineral samples.

\section{Single pulse mode}

For SP imaging, the procedure followed mainly a line-scan protocol used previously. ${ }^{20,24-26}$ The translation stage moved the sample at constant speed along multiple lines while the laser is continuously triggered. This resulted in a rectangular array of edge-to-edge ablation spots of a single ablation layer.
Opposite to the previous protocol, with each laser pulse the imaging control system triggered the ICP-TOFMS to acquire data for a time duration sufficient for the aerosol signal of each individual pulse. The employment of the low dispersion ablation cell in conjunction with the high time resolution of the ICPTOFMS enabled laser pulse rates of $100 \mathrm{~Hz}$ while pulse-to-pulse signal separation of FW0.01M is maintained.

As an example, we used this mode to image the ilvaitehedenbergite assemblage of sample RM3 (Fig. 2). Sample RM3 was imaged over an area of $3000 \mu \mathrm{m} \times 3000 \mu \mathrm{m}$ using the SP mode. The data was acquired in 36 subsections, each one

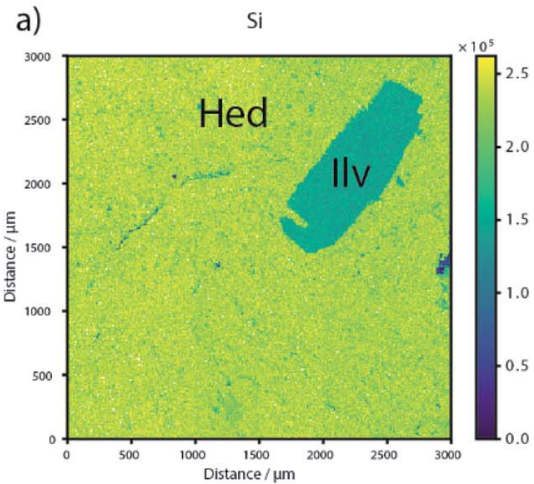

d)

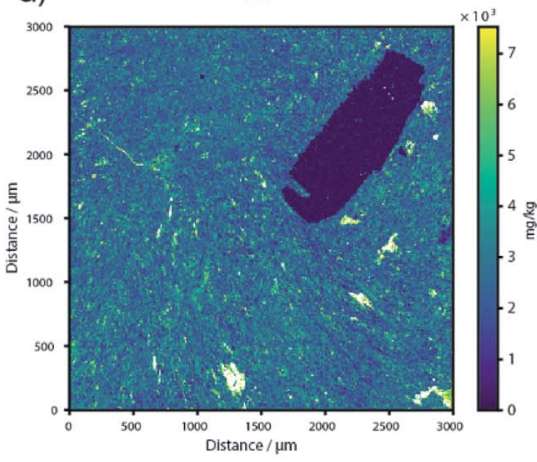

g)

In

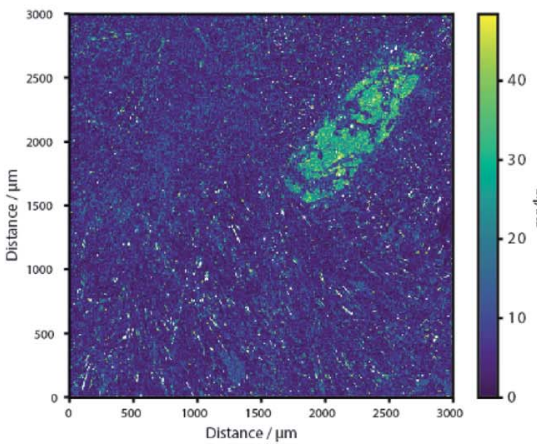

b)

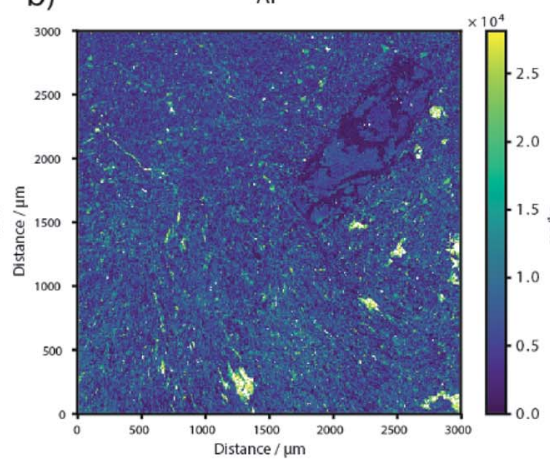

e)

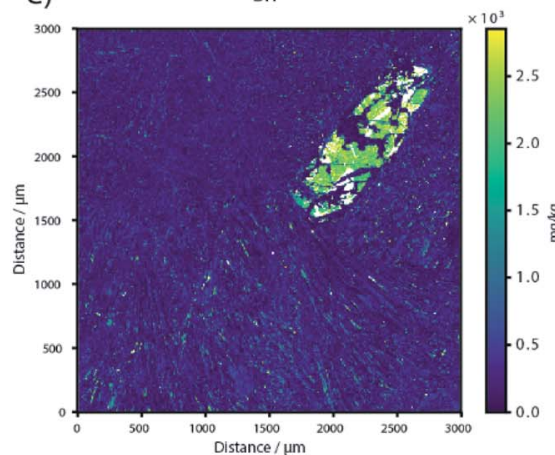

h)

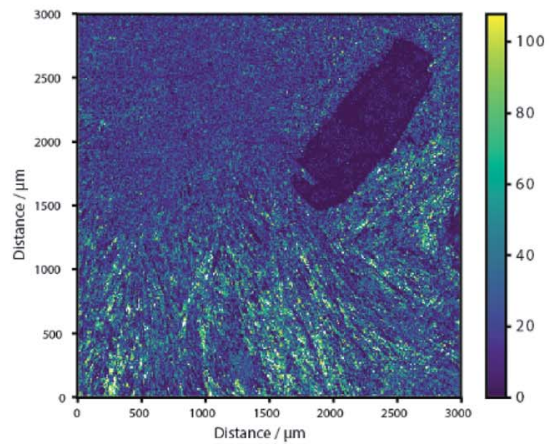

c)

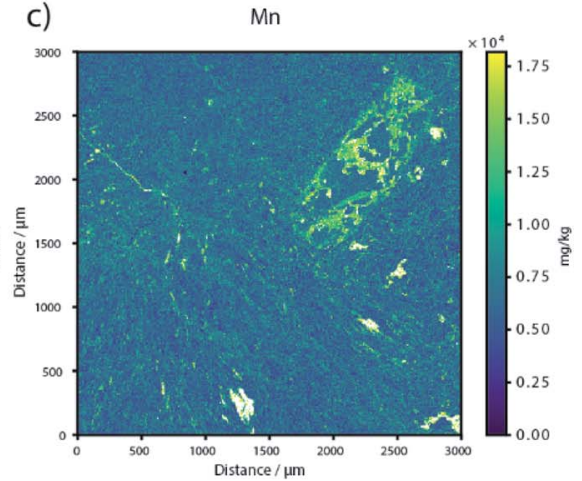

f)

$\mathrm{Rb}$

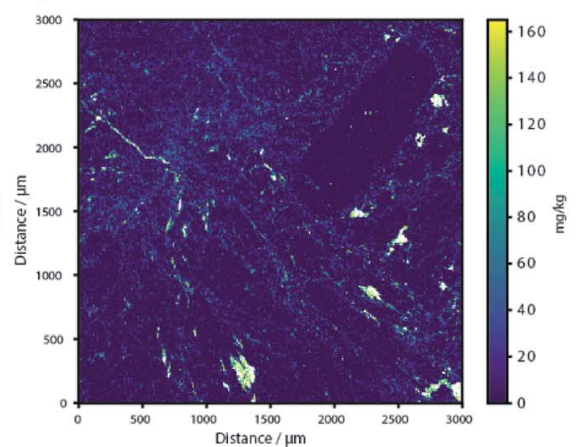

i)

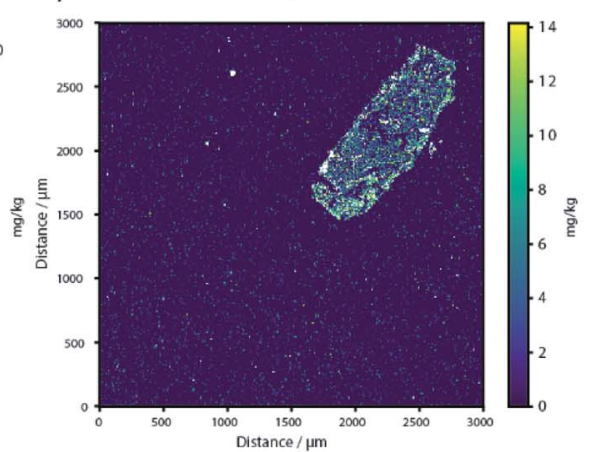

Fig. 2 Element imaging of an ilvaite (Ilv)-hedenbergite (Hed) assemblage of sample RM3 generated with the SP mode. The panels include a representative selection of major $(a-c)$, minor $(d$ and $e$ ) and trace elements ( $f-i)$ of these two minerals, whose lateral distribution identify distinct crystalline morphologies and compositions. $\mathrm{Si}$ (a) is mostly homogeneously distributed within the two minerals, but this is not the case for most major, minor, and trace elements. Ilvaite shows measurable mass fractions of a set of major (Al, Mn), minor (Sn) and trace elements (e.g., In and Dy). However, they are inhomogeneously distributed within the crystal. Notice that the irregular areas having low Al, Sn, and In mass fractions are the mirror image of those showing high Mn mass fractions. This is probably due to the coupled substitutions of these elements in the crystal structure. The aggregate of hedenbergite crystals shows the occurrence of irregular patches having consistently high Al (b), Mn (c), $\mathrm{Na}$ (d) and Rb (f) mass fractions indicate the presence of distinct - previously unidentified phases. Also, Sb (h) is inhomogeneously distributed within hedenbergite. 
covering an area of $500 \mu \mathrm{m} \times 500 \mu \mathrm{m}(100 \times 100$ pixel $) .18$ subsections were acquired automatically and consecutively, and every subsection was triggered in addition to the triggering of each pixel. Reference material NIST SRM 610 was measured for external calibration at the start, after 18 subsections, and after 36 subsections, respectively. The second half of the image (18 subsections) was also acquired automatically. The 36 subsections were combined during the data processing stage.

The measurement time of the area of $3000 \mu \mathrm{m} \times 3000 \mu \mathrm{m}$ was $77 \mathrm{~min}$, resulting in an acquisition speed of approx. $7 \mathrm{~mm}^{2}$ $\mathrm{h}^{-1}$ at $5 \mu \mathrm{m}$ lateral resolution. Considering some communication overhead and the stage movement to the next line start position the effective pixel acquisition rate was 78 pixel per $\mathrm{s}$.

The mass fractions of the majority of rare earth elements (REEs) in the ilvaite crystal such as Sc, La, Ce, Pr, Nd, Sm, Eu, $\mathrm{Gd}, \mathrm{Tb}, \mathrm{Tm}, \mathrm{Yb}$ and Lu were below the LOD. For some REEs such as Y, Dy, Ho, and Er at least small areas containing elevated mass fractions were detected. This shows that while the SP mode cannot provide LODs that are sufficiently low to measure complete distribution maps, but it has ability to determine the distribution of a broad range of components, from major to trace elements which can be well suited for a fast screening of samples.

\section{Hole drilling mode at $100 \mathrm{~Hz}$}

With this ablation mode, a larger sample volume is removed per sampling position following a HD approach without losing lateral resolution. ${ }^{57}$ This is feasible for samples in which multiple pulses per position do not cause total sample consumption (i.e., geological thin sections in contrast to biological thin-tissue sections) and for which the lateral distribution of elements into depth can be assumed similar. However, the time resolution of ICP-TOFMS also allows to monitor changes into depth. To this end, multiple laser pulses without stage movement were applied to the same sample position before the stage was moved to the next neighboring position. In addition, the ICP-TOFMS was triggered for data acquisition once per position for a prolonged time span to acquire not data for individual laser pulses, but a transient signal for multiple pulses without the inherent need to resolve individual signals (Fig. 3). The HD ablation mode in combination with the presented software control approach was already applied successfully to gain insights into other geological processes. ${ }^{58}$

The ablation depth per laser pulse depends largely on the sample composition and is in most cases significantly smaller than $1 \mu \mathrm{m}$ (e.g. NIST SRM $610 \approx 0.2 \mu \mathrm{m}$ per pulse at $15 \mathrm{~J}$ $\left.\mathrm{cm}^{-2}\right) .{ }^{59}$ The burst of 25 laser pulses per sample position resulted in surface sampling diameter of $5 \mu \mathrm{m}$ and a depth of approximately $5 \mu \mathrm{m}$. A larger number of laser pulses per position leads in total to a higher signal intensity per pixel, and therefore to higher pixel sensitivity while maintaining the lateral resolution. The number of laser pulses and time duration for data acquisition can be adjusted for different laser spot sizes or sample composition.

We applied this approach to the ilvaite-quartz assemblage of sample RM2 (Fig. 4). This sample was imaged over an area of
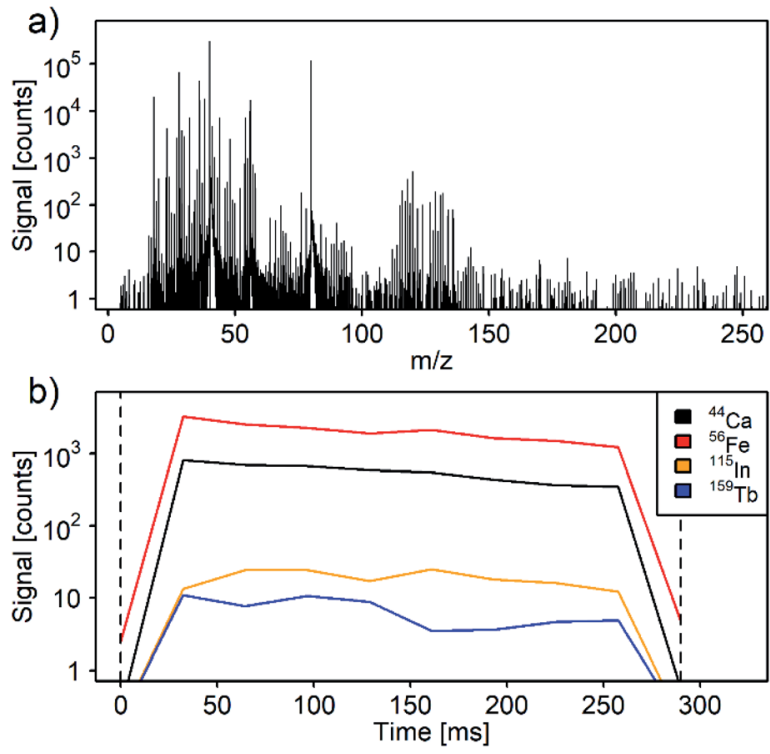

Fig. 3 Full mass spectrum (a) and transient signal (b) are acquired for each sample position. As an example, the signal of a hole drilling imaging measurement of one sample position on ilvaite is displayed (Fig. 4, $x=670 \mu \mathrm{m}, y=395 \mu \mathrm{m}$ ). The transient signal shows homogenous distribution of the elements $\mathrm{Ca}, \mathrm{Fe}, \mathrm{In}$, and $\mathrm{Tb}$ in depth. Due to the lower data acquisition rate for the hole drilling mode, transient signals from individual laser pulses are not distinguishable here (compare with ESI†).

$600 \mu \mathrm{m} \times 1300 \mu \mathrm{m}$. The data was acquired in two subsections of $600 \mu \mathrm{m} \times 650 \mu \mathrm{m}(120 \times 130$ pixel $)$. The measurement of reference material NIST SRM 610 was carried out for external calibration at the beginning and at the end of each subsection acquisition. The two subsections were combined later on, during the data processing stage.

The $600 \mu \mathrm{m} \times 1300 \mu \mathrm{m}$ area measured of sample RM2 took $213 \mathrm{~min}$, resulted in an acquisition speed of approx. $0.22 \mathrm{~mm}^{2}$ $\mathrm{h}^{-1}$ at $5 \mu \mathrm{m}$ lateral resolution. Considering some communication overhead and the stage movement to the next line start position, the effective pixel acquisition rate was 2.4 pixel per $\mathrm{s}$. Further development of the HD imaging procedure allowed to speed up the effective pixel acquisition rate to 2.9-3.0 pixel per $\mathrm{s}$ which was subsequently used for imaging of a shape adapted ablation area. $250 \mathrm{~ms}$ per sample position are required for 25 laser pulses at $100 \mathrm{~Hz}$ and signal detection. In addition, after firing 25 laser pulses the imaging control system required an average of $80-90 \mathrm{~ms}$ to move the stage to the next sample position and to receive the feedback. Compared to the SP mode, the HD mode was 27 times slower.

According to the different applications it is important to note that despite of the slower speed of the HD mode, its lower LOD allowed the determination of trace elements which occur typically in the low $\mathrm{mg} \mathrm{kg}^{-1}$ range. The HD mode enabled quantification of most of the REEs, as well as areas containing elevated mass fractions of Sc, Y, La, Ce, Pr, Nd, Sm, Gd, Tb, Dy, Ho, Er, $\mathrm{Tm}, \mathrm{Yb}, \mathrm{Lu}, \mathrm{Pb}$, Th and $\mathrm{U}$ which enhance the understanding of the crystal formation processes as reported in [e.g., ref. 60 and 
a)

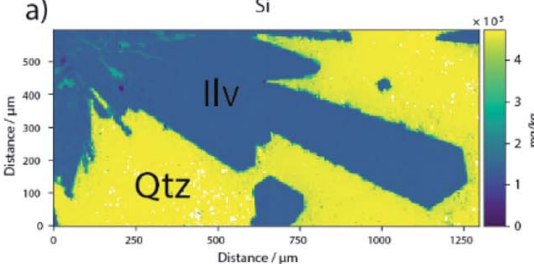

d)

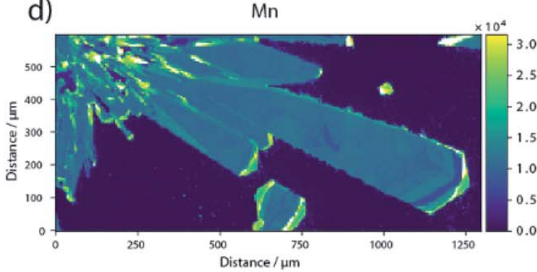

In

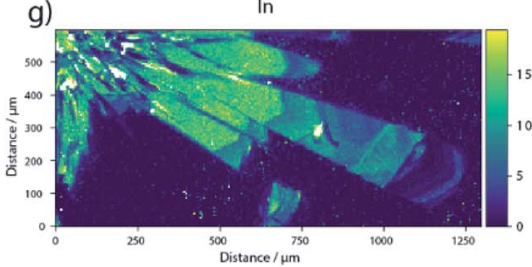

Sm

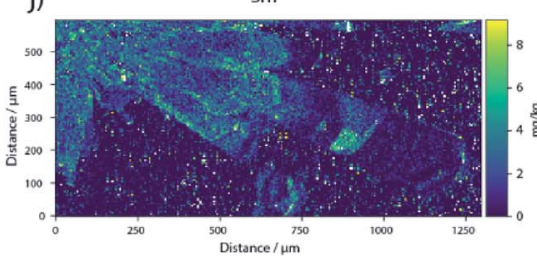

b)

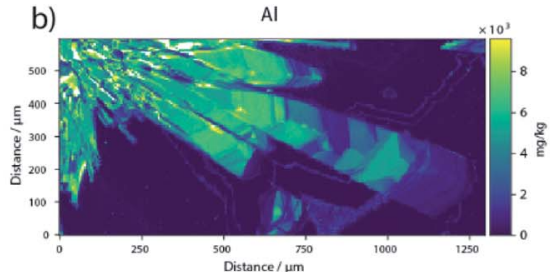

e) $\mathrm{Sn}$

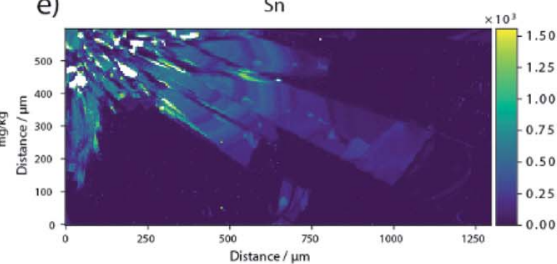

$\mathrm{Sb}$

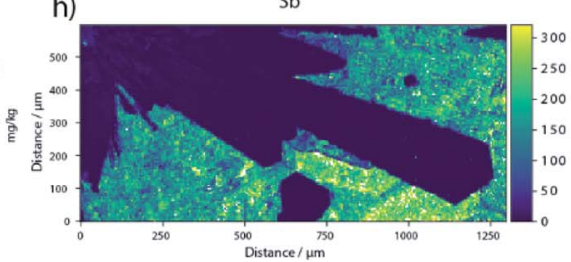

k)

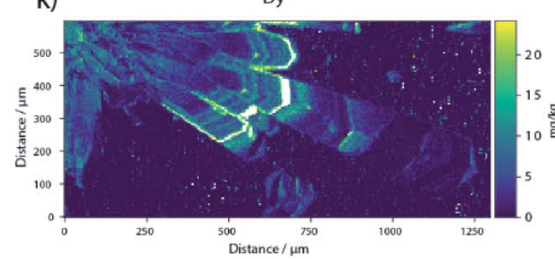

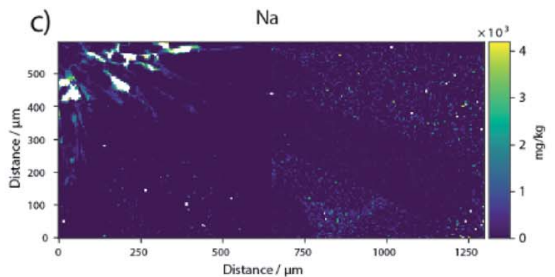

$\mathrm{Rb}$
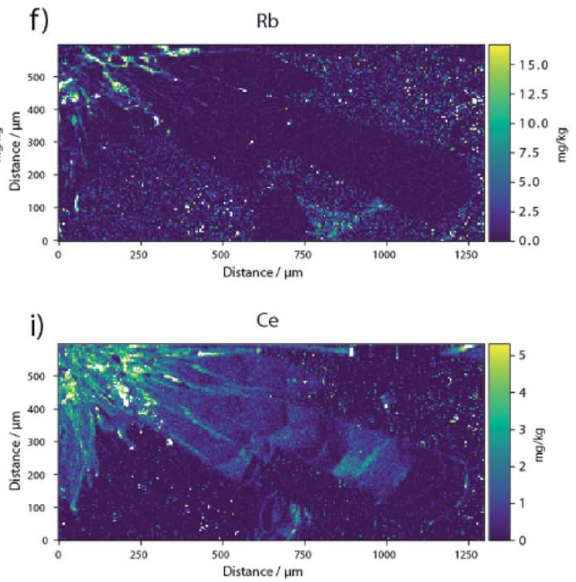

l)

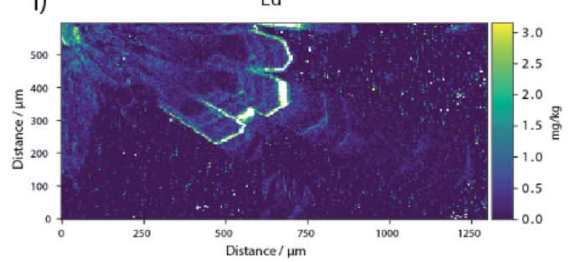

Fig. 4 Element imaging of the ilvaite (Ilv) -quartz (Qtz) assemblage of sample RM2 generated with HD mode. The panels include a representative selection of major ( $a$ and b), minor $(c-e)$ and trace elements $(f-l)$ of these two minerals, whose lateral distributions mark a distinct crystalline texture. Similar to what is displayed for RM3 (Fig. 2), also this sample shows a homogeneous distribution of Si (a), but this is not the case for most major, minor, and trace elements. Within ilvaite, a large number of elements (Al, Mn, Sn, In, REEs, others not shown) mark bands of high and low mass fractions in the elongated prisms that may be 5-10 $\mu \mathrm{m}$ in width. In contrast, quartz does not show a systematic distribution of minor and trace elements with the exception for Al (b), alkali metals (c and f), and Sb (h).

61]. Eu was the only REE whose mass fraction was below the LOD $\left(0.4 \mathrm{mg} \mathrm{kg}^{-1}\right)$.

\section{Comparison of ablation modes}

A comparison of the distinct ablation modes is given in Fig. 5, which was constructed by merging areas next to each other of
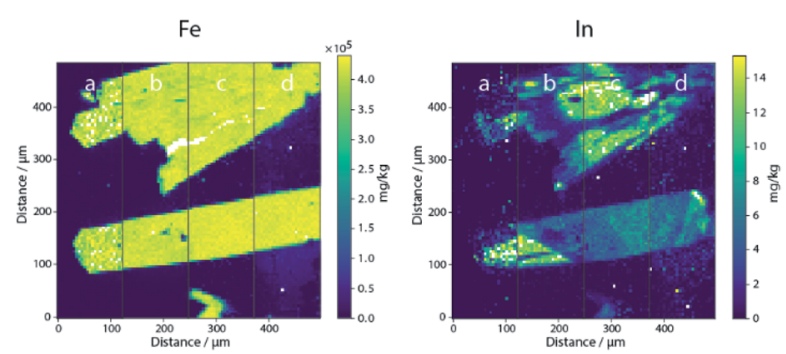

Fig. 5 Quantified maps of Fe and In of an ilvaite-quartz assemblage of sample RM2 comparing the ablation modes SP (a), HD (b), CP + HD (c), and $O L(d)$. Notice that this sample area analyzed is smaller compared to the imaging displayed in Fig. 2 and 4. sample RM2 analyzed by SP, HD, CP + HD, and OL. In addition to imaging speed, the pixel sensitivity, expressed as accumulative counts per unit mass fraction, is a key parameter that can be effectively used to compare different ablation modes due to its impact on image quality and LODs. Summing the signal of 25 laser pulses for HD signal intensity and therefore the pixel sensitivity increased when compared to SP mode. On the other hand, gas blank intensities increased as well due to the longer integration time for the HD mode. However, most of the heavy elements showed low gas blank signals and the LODs for most elements were 10-20 times lower in comparison to the SP mode (Fig. 6 and ESI Table S1†). Therefore, the HD mode allowed the quantification of REEs traces at lower mass fractions. Due to the numerical aperture of the objective, and to the changes of sample surface after the first laser pulse ${ }^{62} 25$ times higher sensitivities were not achieved when summing the signal for 25 laser pulses using the HD mode.

For the same element the pixel sensitivity and consequently the LOD largely depended on the amount of material ablated as laser ablation rates varied depending on the composition of the 


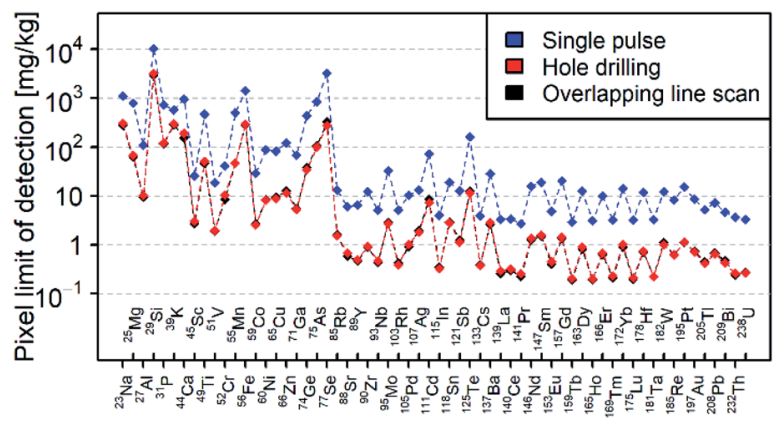

Fig. 6 Pixel LODs for the ablation modes HD, OL and SP using NIST SRM 610 according to Table S1. $\uparrow$ The notch filter was applied at $\mathrm{m} / z 56$ and $\mathrm{m} / \mathrm{z} 75$ leading to higher LODs for elements with close $\mathrm{m} / \mathrm{z}$ isotopes such as $\mathrm{Mn}, \mathrm{Fe}, \mathrm{Ga}, \mathrm{Ge}, \mathrm{As}$, and Se. Data points of $\mathrm{OL}$ are covered by them of HD as the LODs for HD and OL are similar.

material of interest..$^{59}$ To compare the ablation rate of NIST SRM 610 , ilvaite, hedenbergite, and quartz the ${ }^{29} \mathrm{Si}$ pixel sensitivity was used. The composition of each mineral was calculated based on its stoichiometric formula. The pixel sensitivity for ilvaite and hedenbergite was in a range of $\pm 15 \%$ compared to NIST SRM 610. Therefore, we assumed a similar ablation rate for ilvaite, hedenbergite and NIST SRM 610 as well as similar LODs. However, the pixel sensitivity for quartz was in average $60-70 \%$ lower than that of NIST SRM 610 resulting in approximately three times higher LODs compared to NIST SRM 610. This is assumed to be caused by a three times lower ablation rate of quartz.

The lower LODs obtained from the HD mode allowed measuring the composition of different minerals in great detail. For example, HD applied to ilvaite permitted to detect the distribution of In, whereas the SP mode creates a blurred distribution to the point that the edges of the ilvaite crystals were barely visible (LOD In: SP $4 \mathrm{mg} \mathrm{kg}^{-1}$, HD $0.3 \mathrm{mg} \mathrm{kg}^{-1}$, OL $0.3 \mathrm{mg} \mathrm{kg}^{-1}$ ).

The HD mode can also be used with an additional cleaning pulse (CP + HD) at each sampling position prior to hole drilling and data acquisition, which is specifically important when crossing phases or when re-deposition of ablated material occurs. ${ }^{48}$ To this end, a single cleaning pulse was applied by triggering the laser without ICP-TOFMS data acquisition. For this sample, an influence of surface contamination and the resulting element map was not observed. The CP + HD mode allowed an effective acquisition speed of 1.9-2.0 pixel per s. Further optimization of the $\mathrm{CP}+\mathrm{HD}$ imaging procedure similar to the improvements in the HD mode allowed to speed up the effective pixel acquisition speed to 2.8-2.9 pixel per s.

The imaging control system allows also an overlapping line scan (OL) mode where each laser pulse can be acquired separately or as reported here summing the signal of 25 laser pulses to lower the LOD. The OL mode delivered LODs that were indistinguishable from those of the HD mode (Fig. 6) and an effective acquisition speed of 3.8-3.9 pixel per s, but was different in the effective resolution compared to the HD mode. The resolution of the OL mode in the line scan direction was slightly lower than that of the HD mode. This was caused by the overlap of the ablation spots of consecutive pixels. However, we stress that no data deconvolution was applied in our maps. ${ }^{31,33}$

\section{Geological information}

Samples RM2 and RM3 show relatively homogeneous optical properties and homogeneous compositions in terms of major element mass fractions. However, elemental imaging of a large number of elements captures unexpected compositional features at the scale of individual crystals and whole sample. In the RM3 sample analyzed by the SP mode (Fig. 2), the coupled variations in mass fraction of major, minor, and trace elements within ilvaite suggest a complex crystallization history. This evidence alone represents a relevant set of compositional information, which constrains mineral formation to a great extent. Thus, the inhomogeneous distribution of $\mathrm{Al}, \mathrm{Sn}$, In, and in part REEs in the ilvaite crystal is the mirror image of that of Mn (Fig. 2), which indicates that the texture visible today for RM3 must have formed after a two-step crystallization event. The first step formed the relatively high mass fractions of Al, Sn, and In in the crystal, while the second step caused the coupled $\mathrm{Mn}$ enrichment and $\mathrm{Al}-\mathrm{Sn}-\mathrm{In}$ depletion as a result of microfracturing, dissolution, and recrystallization of the previous crystal. ${ }^{63}$ The relatively fast ablation involved by the SP mode onto the RM3 sample cannot provide LODs that are sufficiently low to determine the mass fractions of the entire set of trace and ultra-trace elements as most REEs. Hence, the SP mode proves being a fast screening method to highlight the key compositional and textural features of geological samples.

The RM2 sample analyzed with the HD mode (Fig. 4) discloses lateral distributions of major, minor, and trace elements that are variable at the scale of $10 \mu \mathrm{m}$ or less (1-2 pixels) and that were captured for mass fraction differences in the order of few $\mathrm{mg} \mathrm{kg}^{-1}$ in neighboring areas of the crystals. This was shown by the relatively subtle changes of $\mathrm{Al}, \mathrm{Mn}, \mathrm{Sn}$, In, and REEs mass fractions within ilvaite, which form alternating high and low mass fraction bands of 5-10 $\mu \mathrm{m}$ in width (Fig. 4). Notably, the distributions of Al, $\mathrm{Sb}$, and alkali metals mass fractions within the quartz of RM2 indicate also relatively subtle changes. There were also thin and irregular bright bands within ilvaite corresponding to high $\mathrm{Al}, \mathrm{Mn}$, $\mathrm{Na}, \mathrm{Sn}, \mathrm{Rb}, \mathrm{In}$, and Ce mass fractions.

In summary, the element distribution maps constructed with the HD mode were able to capture multiple stages of mineral growth in geological samples, with which the lateral distribution of a large number of major, minor, and trace elements could be determined at low LODs. This gamut of very detailed compositional variations reflects the superior pixel sensitivity of the HD approach, which provided deeper insights into the crystallization histories of the solids.

\section{Shape adapted ablation area}

In most ablation experiments, a square or rectangle is not the shape of the ROI that is actually intended to be studied in a sample. Thus, the use of geometrically regular ablation pattern leaves a significant portion of the measurement time not effectively used. To overcome this limitation, an imaging 

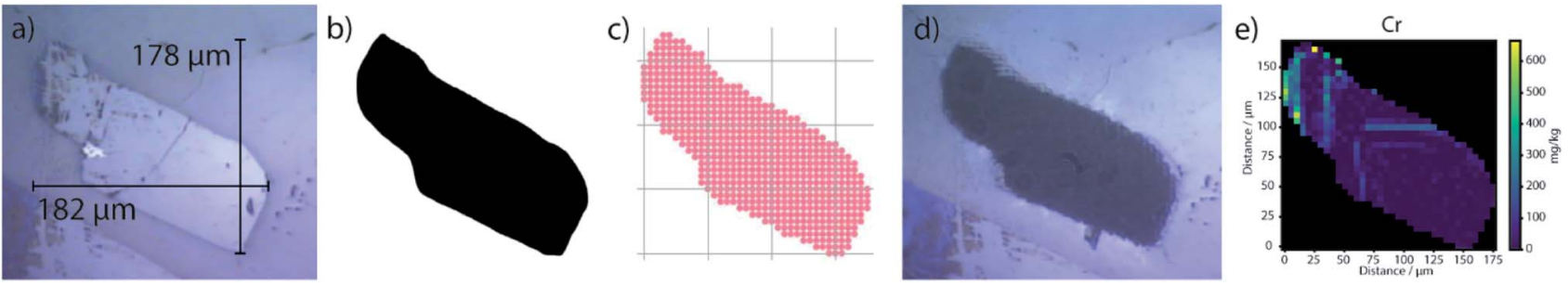

Fig. 7 Different stages for a shape adapted ablation area experiment of an ilvaite crystal with $5 \mu$ m spot size. (a) Photomicrograph of sample taken with the petrographic microscope (reflected-light image, parallel nicols) and definition of size, (b) black and white drawing of the crystal, (c) calculated ablation spot array, (d) ablated crystal, (e) quantified $\mathrm{Cr}$ distribution map showing internal texture of the mineral.

control software enabling a shape adapted sample ablation was developed.

To determine the effective area for analysis, a photomicrograph of the ROI of the sample is recorded and the size of the desired ablation area is measured in two orthogonal $x-y$ directions. A black and white overlay is created using the photomicrograph. On a later step the actual ROI is dimensioned using the overlay, and its image is extracted from the surrounding area. Using the black and white overlay, the imaging control software calculates the ablation positions of the identified area, the laser spot size, and the image dimensions in the $x$ and $y$ directions. The data evaluation program uses this virtual mask to link the pixel data with the relative ablation positions, and generates the element distribution maps (Fig. 7). The shape adapted sample ablation was applied in a stepped sampling mode, during which the sample stage movement is controlled to enable precise positioning and is limited by the speed of the stage moving and by the feedback cycle. Therefore, a comparison was only made for the HD mode.

The ablation area fitted to the mineral shape enabled a reduced data acquisition time in this example by a factor of 2 compared to what would have been necessary by the imaging of a rectangular area of the same crystal in HD mode (605 pixels compared to 1332 pixels). The presented imaging of shape adapted ablation areas turned out particularly useful for small, non-rectangular or non-circular and even vein-like ROI, which could then be analyzed fit-to-shape.

The generation of composite spectra and further quantification of elements based on those spectra, and specific mineral assignments, are possible similar to the 'inspector' functionalities presented by Petrus et al. in the 'Monocle' interrogation software tool. ${ }^{44}$

\section{Conclusion}

We report a combination of an attuned and automated, software-controlled, customized imaging control system for LAICP-TOFMS. The system allows adaptable ablation modes (via single pulse or hole drilling (with and without cleaning of the surface)) which significantly improves elemental imaging of mineral samples. The various ablation modes provide access to task-specific detection capabilities which is important for accurate determination of major, minor, and trace element distributions.
Our data demonstrates that the single pulse ablation mode is well suited for fast imaging of major, minor, and a limited set of trace elements in mineral samples. Hole drilling imaging provides an order of magnitude improved LODs for most elements at constant lateral resolution. Therefore, the HD mode provides a more representative sampling result and can be considered as the method of choice for accurate quantification of a large number of major to trace elements in minerals. Furthermore, the fast data acquisition allows monitoring into depth to reveal multi-phase sampling.

We show further that a significant time saving step for elemental imaging could be the use of shape adapted ablation areas, which reduce the effective ablated area and therefore the duration of data acquisition. Triggering and binning of each ablation position allows to link the pixel data with the relative sample position and simplifies data evaluation and image generation.

\section{Conflicts of interest}

There are no conflicts to declare.

\section{Acknowledgements}

The authors would like to thank Jonas Windhager (University Zurich) for his contribution in software development and Roland Mäder from the ETH workshop (ETH Zurich) for construction of the modified ablation cell. Marcel Burger, Alexander Gundlach-Graham, and Bodo Hattendorf are gratefully acknowledged for helpful discussions.

\section{References}

$1 \mathrm{~V}$. M. Goldschmidt, The principles of distribution of chemical elements in minerals and rocks. The seventh Hugo Müller Lecture, delivered before the Chemical Society on March 17th, 1937, J. Chem. Soc., 1937, 655-673.

2 B. Mason, Minor and trace element distribution in minerals of the Muzzle River Gabbro, N. Z. J. Geol. Geophys., 1972, 15(3), 465-475.

3 C. J. Allègre, M. Treuil, J.-F. Minster, B. Minster and F. Albarède, Systematic use of trace element in igneous process, Contrib. Mineral. Petrol., 1977, 60(1), 57-75. 
4 K. D. Putirka, Thermometers and Barometers for Volcanic Systems, Rev. Mineral. Geochem., 2008, 69(1), 61-120.

5 S. K. Saxena, Distribution of elements in coexisting minerals and the problem of chemical disequilibrium in metamorphosed basic rocks, Contrib. Mineral. Petrol., 1969, 20(2), 177-197.

6 E. F. Baxter, M. J. Caddick and J. J. Ague, Garnet: Common Mineral, Uncommonly Useful, Elements, 2013, 9(6), 415-419.

7 D. Gagnevin, J. S. Daly and A. Kronz, Zircon texture and chemical composition as a guide to magmatic processes and mixing in a granitic environment and coeval volcanic system, Contrib. Mineral. Petrol., 2010, 159(4), 579-596.

8 C. Dupuis and G. Beaudoin, Discriminant diagrams for iron oxide trace element fingerprinting of mineral deposit types, Miner. Deposita, 2011, 46(4), 319-335.

9 R. S. Houk, V. A. Fassel, G. D. Flesch, H. J. Svec, A. L. Gray and C. E. Taylor, Inductively coupled argon plasma as an ion source for mass spectrometric determination of trace elements, Anal. Chem., 1980, 52(14), 2283-2289.

10 A. L. Gray, Solid sample introduction by laser ablation for inductively coupled plasma source mass spectrometry, Analyst, 1985, 110(5), 551-556.

11 R. J. Reeder and J. C. Grams, Sector zoning in calcite cement crystals: implications for trace element distributions in carbonates, Geochim. Cosmochim. Acta, 1987, 51(2), 187-194.

12 D. J. Sinclair, L. P. J. Kinsley and M. T. McCulloch, High resolution analysis of trace elements in corals by laser ablation ICP-MS, Geochim. Cosmochim. Acta, 1998, 62(11), 1889-1901.

13 C. B. Chernoff and W. D. Carlson, Trace element zoning as a record of chemical disequilibrium during garnet growth, Geology, 1999, 27(6), 555-558.

14 C. I. Prince, J. Kosler, D. Vance and D. Günther, Comparison of laser ablation ICP-MS and isotope dilution REE analyses-implications for Sm-Nd garnet geochronology, Chem. Geol., 2000, 168(3), 255-274.

15 M. Sanborn and K. Telmer, The spatial resolution of LA-ICPMS line scans across heterogeneous materials such as fish otoliths and zoned minerals, J. Anal. At. Spectrom., 2003, 18(10), 1231-1237.

16 J. Woodhead, S. Swearer, J. Hergt and R. Maas, In situ Srisotope analysis of carbonates by LA-MC-ICP-MS: interference corrections, high spatial resolution and an example from otolith studies, J. Anal. At. Spectrom., 2005, 20(1), 22-27.

17 J. D. Woodhead, J. Hellstrom, J. M. Hergt, A. Greig and R. Maas, Isotopic and Elemental Imaging of Geological Materials by Laser Ablation Inductively Coupled PlasmaMass Spectrometry, Geostand. Geoanal. Res., 2007, 31(4), 331-343.

18 J. S. Becker, M. V. Zoriy, C. Pickhardt, N. Palomero-Gallagher and K. Zilles, Imaging of Copper, Zinc, and Other Elements in Thin Section of Human Brain Samples (Hippocampus) by Laser Ablation Inductively Coupled Plasma Mass Spectrometry, Anal. Chem., 2005, 77(10), 3208-3216.

19 R. W. Hutchinson, A. G. Cox, C. W. McLeod, P. S. Marshall, A. Harper, E. L. Dawson and D. R. Howlett, Imaging and spatial distribution of $\beta$-amyloid peptide and metal ions in Alzheimer's plaques by laser ablation-inductively coupled plasma-mass spectrometry, Anal. Biochem., 2005, 346(2), 225-233.

20 A. Gundlach-Graham, M. Burger, S. Allner, G. Schwarz, H. A. O. Wang, L. Gyr, D. Grolimund, B. Hattendorf and D. Günther, High-Speed, High-Resolution, Multielemental Laser Ablation-Inductively Coupled Plasma-Time-of-Flight Mass Spectrometry Imaging: Part I. Instrumentation and Two-Dimensional Imaging of Geological Samples, Anal. Chem., 2015, 87(16), 8250-8258.

$21 \mathrm{~J}$. S. Becker, Imaging of metals in biological tissue by laser ablation inductively coupled plasma mass spectrometry (LA-ICP-MS): state of the art and future developments, $J$. Mass Spectrom., 2013, 48(2), 255-268.

22 C. Giesen, H. A. O. Wang, D. Schapiro, N. Zivanovic, A. Jacobs, B. Hattendorf, P. J. Schüffler, D. Grolimund, J. M. Buhmann, S. Brandt, Z. Varga, P. J. Wild, D. Günther and B. Bodenmiller, Highly multiplexed imaging of tumor tissues with subcellular resolution by mass cytometry, Nat. Methods, 2014, 11, 417.

23 D. J. Hare, J. L. George, R. Grimm, S. Wilkins, P. A. Adlard, R. A. Cherny, A. I. Bush, D. I. Finkelstein and P. Doble, Three-dimensional elemental bio-imaging of $\mathrm{Fe}, \mathrm{Zn}, \mathrm{Cu}$, $\mathrm{Mn}$ and $\mathrm{P}$ in a 6-hydroxydopamine lesioned mouse brain, Metallomics, 2010, 2(11), 745-753.

24 M. Burger, A. Gundlach-Graham, S. Allner, G. Schwarz, H. A. Wang, L. Gyr, S. Burgener, B. Hattendorf, D. Grolimund and D. Gunther, High-speed, highresolution, multielemental LA-ICP-TOFMS imaging: part II. Critical evaluation of quantitative three-dimensional imaging of major, minor, and trace elements in geological samples, Anal. Chem., 2015, 87(16), 8259-8267.

25 M. Burger, G. Schwarz, A. Gundlach-Graham, D. Kaser, B. Hattendorf and D. Gunther, Capabilities of laser ablation inductively coupled plasma time-of-flight mass spectrometry, J. Anal. At. Spectrom., 2017, 32(10), 1946-1959. 26 A. Gundlach-Graham, P. S. Garofalo, G. Schwarz, D. Redi and D. Günther, High-resolution, Quantitative Element Imaging of an Upper Crust, Low-angle Cataclasite (Zuccale Fault, Northern Apennines) by Laser Ablation ICP Time-of-Flight Mass Spectrometry, Geostand. Geoanal. Res., 2018, 42(4), 559-574.

27 O. Borovinskaya, B. Hattendorf, M. Tanner, S. Gschwind and D. Gunther, A prototype of a new inductively coupled plasma time-of-flight mass spectrometer providing temporally resolved, multi-element detection of short signals generated by single particles and droplets, J. Anal. At. Spectrom., 2013, 28(2), 226-233.

28 H. A. Wang, D. Grolimund, C. Giesen, C. N. Borca, J. R. ShawStewart, B. Bodenmiller and D. Gunther, Fast chemical imaging at high spatial resolution by laser ablation inductively coupled plasma mass spectrometry, Anal. Chem., 2013, 85(21), 10107-10116.

29 S. J. M. Van Malderen, T. Van Acker and F. Vanhaecke, Submicrometer Nanosecond LA-ICP-MS Imaging at Pixel 
Acquisition Rates above $250 \mathrm{~Hz}$ via a Low-Dispersion Setup, Anal. Chem., 2020, 92(8), 5756-5764.

30 Y. Ke, J. Zhou, L. Qiao, M. Zhang, W. Guo, L. Jin and S. Hu, High spatial resolution quantitative elemental imaging of foraminifer by laser ablation-inductively coupled plasmamass spectrometry, Anal. Methods, 2019, 11(16), 2129-2137.

31 A. Plotnikov, C. Vogt and K. Wetzig, An approach to the reconstruction of true concentration profile from transient signal in spatially resolved analysis by means of laser ablation ICP MS, J. Anal. At. Spectrom., 2002, 17(9), 11141120.

32 D. Drescher, C. Giesen, H. Traub, U. Panne, J. Kneipp and N. Jakubowski, Quantitative Imaging of Gold and Silver Nanoparticles in Single Eukaryotic Cells by Laser Ablation ICP-MS, Anal. Chem., 2012, 84(22), 9684-9688.

33 S. J. M. Van Malderen, J. T. van Elteren and F. Vanhaecke, Submicrometer Imaging by Laser Ablation-Inductively Coupled Plasma Mass Spectrometry via Signal and Image Deconvolution Approaches, Anal. Chem., 2015, 87(12), 6125-6132.

34 J. Pisonero, D. Bouzas-Ramos, H. Traub, B. Cappella, C. Álvarez-Llamas, S. Richter, J. C. Mayo, J. M. CostaFernandez, N. Bordel and N. Jakubowski, Critical evaluation of fast and highly resolved elemental distribution in single cells using LA-ICP-SFMS, J. Anal. At. Spectrom., 2019, 34(4), 655-663.

35 K. Löhr, H. Traub, A. J. Wanka, U. Panne and N. Jakubowski, Quantification of metals in single cells by LA-ICP-MS: comparison of single spot analysis and imaging, J. Anal. At. Spectrom., 2018, 33(9), 1579-1587.

36 A. Gundlach-Graham and D. Günther, Toward faster and higher resolution LA-ICPMS imaging: on the co-evolution of LA cell design and ICPMS instrumentation, Anal. Bioanal. Chem., 2016, 408(11), 2687-2695.

37 D. N. Douglas, A. J. Managh, H. J. Reid and B. L. Sharp, HighSpeed, Integrated Ablation Cell and Dual Concentric Injector Plasma Torch for Laser Ablation-Inductively Coupled Plasma Mass Spectrometry, Anal. Chem., 2015, 87(22), 11285-11294.

38 S. J. M. Van Malderen, J. T. van Elteren and F. Vanhaecke, Development of a fast laser ablation-inductively coupled plasma-mass spectrometry cell for sub-[small mu ]m scanning of layered materials, J. Anal. At. Spectrom., 2015, 30(1), 119-125.

39 M. Tanner and D. Günther, In torch laser ablation sampling for inductively coupled plasma mass spectrometry, J. Anal. At. Spectrom., 2005, 20(9), 987-989.

40 B. Paul, J. D. Woodhead, C. Paton, J. M. Hergt, J. Hellstrom and C. A. Norris, Towards a Method for Quantitative LAICP-MS Imaging of Multi-Phase Assemblages: Mineral Identification and Analysis Correction Procedures, Geostand. Geoanal. Res., 2014, 38(3), 253-263.

41 R. Weiskirchen, S. Weiskirchen, P. Kim and R. Winkler, Software solutions for evaluation and visualization of laser ablation inductively coupled plasma mass spectrometry imaging (LA-ICP-MSI) data: a short overview, J. Cheminf., 2019, 11(1), 16.
42 C. Paton, J. Hellstrom, B. Paul, J. Woodhead and J. Hergt, Iolite: freeware for the visualisation and processing of mass spectrometric data, J. Anal. At. Spectrom., 2011, 26(12), 2508-2518.

43 B. Paul, C. Paton, A. Norris, J. Woodhead, J. Hellstrom, J. Hergt and A. Greig, CellSpace: a module for creating spatially registered laser ablation images within the Iolite freeware environment, J. Anal. At. Spectrom., 2012, 27(4), 700-706.

44 J. A. Petrus, D. M. Chew, M. I. Leybourne and B. S. Kamber, A new approach to laser-ablation inductively-coupled-plasma mass-spectrometry (LA-ICP-MS) using the flexible map interrogation tool 'Monocle', Chem. Geol., 2017, 463, 76-93.

45 T. Suzuki, S. Sakata, Y. Makino, H. Obayashi, S. Ohara, K. Hattori and T. Hirata, iQuant2: software for Rapid and Quantitative Imaging Using Laser Ablation-ICP Mass Spectrometry, Mass Spectrom., 2018, 7(1), A0065.

46 A. J. Managh and P. Reid, A new freeware tool for image processing and its application to high speed LA-ICP-MS imaging, J. Anal. At. Spectrom., 2019, 34(7), 1369-1373.

47 C. Neff, J. Koch, M. Burger, G. Schwarz, and D. Günther, Modified low aerosol dispersion ablation cell for fast LAICP-TOFMS imaging, manuscript in preparation.

48 S. M. Eggins, L. P. J. Kinsley and J. M. G. Shelley, Deposition and element fractionation processes during atmospheric pressure laser sampling for analysis by ICP-MS, Appl. Surf. Sci., 1998, 127-129, 278-286.

49 Technology, N. I. o. S., Certificate of Analysis: Standard Reference Material 610, 2012.

50 K. P. Jochum, U. Weis, B. Stoll, D. Kuzmin, Q. Yang, I. Raczek, D. E. Jacob, A. Stracke, K. Birbaum, D. A. Frick, D. Günther and J. Enzweiler, Determination of Reference Values for NIST SRM 610-617 Glasses Following ISO Guidelines, Geostand. Geoanal. Res., 2011, 35(4), 397-429.

51 Y. Liu, Z. Hu, S. Gao, D. Günther, J. Xu, C. Gao and H. Chen, In situ analysis of major and trace elements of anhydrous minerals by LA-ICP-MS without applying an internal standard, Chem. Geol., 2008, 257(1), 34-43.

52 L. A. Currie, Limits for qualitative detection and quantitative determination. Application to radiochemistry, Anal. Chem., 1968, 40(3), 586-593.

53 L. Hendriks, A. Gundlach-Graham, B. Hattendorf and D. Günther, Characterization of a new ICP-TOFMS instrument with continuous and discrete introduction of solutions, J. Anal. At. Spectrom., 2017, 32(3), 548-561.

54 . Team, R. D. C., R: A Language and Environment for Statistical Computing, R Foundation for Statistical Computing, 2020.

55 Team, R., RStudio: Integrated Development for R, RStudio, Inc, 2019.

56 P. Lanari, O. Vidal, V. De Andrade, B. Dubacq, E. Lewin, E. G. Grosch and S. Schwartz, XMapTools: a MATLAB@based program for electron microprobe X-ray image processing and geothermobarometry, Comput. Geosci., 2014, 62, 227-240.

57 C. J. M. Lawley, R. A. Creaser, S. E. Jackson, Z. Yang, B. J. Davis, S. J. Pehrsson, B. Dubé, P. Mercier-Langevin and D. Vaillancourt, Unraveling the Western Churchill 
Province Paleoproterozoic Gold Metallotect: Constraints from Re-Os Arsenopyrite and U-Pb Xenotime Geochronology and LA-ICP-MS Arsenopyrite Trace Element Chemistry at the BIF-Hosted Meliadine Gold District, Nunavut, Canada, Econ. Geol., 2015, 110(6), 1425-1454.

58 D. Rubatto, M. Burger, P. Lanari, B. Hattendorf, G. Schwarz, C. Neff, P. Keresztes Schmidt, J. Hermann, A. Vho and D. Günther, Identification of growth mechanisms in metamorphic garnet by high-resolution trace element mapping with LA-ICP-TOFMS, Contrib. Mineral. Petrol., 2020, 175(7), 61.

59 I. Horn, M. Guillong and D. Günther, Wavelength dependant ablation rates for metals and silicate glasses using homogenized laser beam profiles-implications for LAICP-MS, Appl. Surf. Sci., 2001, 182(1), 91-102.
60 D. Rubatto, Zircon trace element geochemistry: partitioning with garnet and the link between $\mathrm{U}-\mathrm{Pb}$ ages and metamorphism, Chem. Geol., 2002, 184(1-2), 123-138.

$61 \mathrm{H}$. Guo and A. Audétat, Transfer of volatiles and metals from mafic to felsic magmas in composite magma chambers: an experimental study, Geochim. Cosmochim. Acta, 2017, 198, 360-378.

62 O. V. Borisov, X. Mao and R. E. Russo, Effects of crater development on fractionation and signal intensity during laser ablation inductively coupled plasma mass spectrometry, Spectrochim. Acta, Part B, 2000, 55(11), 16931704.

63 J. R. Craig and D. J. Vaughan, Ore mineral textures, in Ore microscopy and ore petrography, John Wiley \& Sons, Inc., 1984, pp. 120-163. 